Denise Sese, MD

Department of Pulmonary Medicine,

Respiratory Institute, Cleveland Clinic
Ahed Makhoul, MD

Department of Hematology and Medical

Oncology, Taussig Cancer Institute,

Cleveland Clinic
Laura Hoeksema, MD

Department of Palliative and Supportive Care,

Taussig Cancer Institute, Cleveland Clinic
Laura Shoemaker D0, MS, FAAHPM

Department of Palliative and Supportive Care,

Taussig Cancer Institute, Cleveland Clinic

\title{
The role of palliative care in COVID-19
}

\section{Posted May 19, 2020}

\section{ABSTRACT}

In light of the COVID-19 pandemic, many healthcare systems are experiencing an increased demand for palliative care (PC). To meet this challenge, the $P C$ team at Cleveland Clinic designed an enterprise-wide response plan organized around 4 domains: staff (educational resources and tools), stuff (medications and supplies), space (recommendations for optimizing physical space and facilities), and systems to facilitate high-quality PC delivery to patients. To mitigate isolation during end-oflife care, the Clinic offers "compassionate exceptions" to strict visitation policies, provides personal protective equipment to visitors of these patients, and facilitates virtual visitation via electronic devices.

\section{INTRODUCTION}

"The relief of suffering and the cure of disease must be seen as twin obligations of a medical profession that is truly dedicated to the care of the sick."

Relieving suffering caused by serious illness, regardless of the anticipated outcome, is the shared responsibility of all clinicians. "Total pain," a term coined by Dame Cicely Saunders, is the concept that pain and suffering have physical, psychological, social, and spiritual components. ${ }^{2}$ Palliative care (PC) is specialized medical care for people living with serious illness. It is focused on providing relief from related symptoms with the goal of improving quality-of lifefor both the patient and the family. PC also plays an essential role in relieving the suffering of patients when they receive disease-directed therapy, when there is hope for recovery, and during end-of-life care when the sole goal is comfort.

"Primary PC" can be provided by any skilled clinician of any specialty. Engaging a PC specialist offers

The statements and opinions expressed in COVID-19 Curbside Consults are based on experience and the available literature as of the date posted. While we try to regularly update this content, any offered recommendations cannot be substituted for the clinical judgment of clinicians caring for individual patients.

doi:10.3949/ccjm.87a.ccc036 an added layer of expertise to assist with the most challenging aspects of serious illness, such as uncontrolled symptoms, complex communication, and decision-making. It is especially important during humanitarian crises because they cause large-scale, multidimensional suffering., ${ }^{3,4}$

\section{PRIMARY AND SPECIALTY PALLIATIVE CARE}

The primary PC provider manages basic symptoms of a serious illness (eg, pain, anxiety, and depression), facilitates discussions (about prognosis, code status, treatment goals, and suffering), ${ }^{5}$ facilitates hospice transitions, and delivers end-of-life care to actively dying patients.

The call for PC specialists emerged in the 1990s, with a national focus on expanding PC teaching to all clinicians, including medical students and residents. Even then, the demand for skills beyond what the primary PC practitioner could provide was high. The PC specialist possesses skills essential for ameliorating more complex or refractory symptoms, extending to grief and existential distress, and for assisting complicated cases of care "futility" or those that include conflict between family members, treatment teams, or both. ${ }^{5}$

\section{PANDEMIC PALLIATIVE CARE}

During the COVID-19 pandemic, clinicians have been forced to provide patient care differently. For example, when treating patients with COVID-19, clinicians must don/doff personal protective equipment (PPE) and consider PPE stewardship, both of which are potential barriers to frequent symptom assessment and serious illness conversations. COVID-19, in particular, has changed care for patients with and without COVID-19. With restrictions on family visitation and in-person interdisciplinary team meetings, clinicians are further challenged to manage their patients' symptoms and complex communication issues.

Healthcare providers must leverage technology for these conversations with patients, their families, and their proxies. Finding ways to develop rapport 
and convey empathy within these technological constraints is a new skillset for many. In light of these changes and challenges, the role of the PC specialist is two-fold: (1) help mitigate the burden of the crisis by providing direct clinical care, including symptom management, serious illness discussions, advance care planning, and psychosocial support to patients and families ${ }^{4,6}$ and (2) help prepare healthcare systems and non-PC colleagues to handle the expanded demand for high-quality PC during crises. ${ }^{7}$ This may be accomplished in a variety of ways, including peer education and support as well as engagement with institutional crisis response teams to ensure PC needs are incorporated into system-level response plans.

\section{PALLIATIVE CARE: OUR RESPONSE AT CLEVELAND CLINIC}

The PC team at Cleveland Clinic, in collaboration with institutional leadership and strategic clinical partners, has designed an enterprise-wide response plan to COVID-19. This strategy aims to ensure high-quality PC delivery for patients and their loved ones and attend to the needs of non-PC clinicians as they care for patients with COVID-19. A model developed in 2008 by the US Task Force on Mass Casualty Critical Care and used during the H1N1 influenza outbreak in 2009 helped shape our current responses to COVID-19.7,8 This model divides disaster response into four domains: staff, stuff, space, and systems (Table 1 ).

\section{DEATH AND DYING WITH COVID-19}

Strict visitation policies and high risk of disease transmission make caring for patients dying with COVID19 difficult. Many care givers are dismayed at the profound isolation that COVID-19 has caused within healthcare settings, especially when considering the impact of this isolation during end-of-life care. The unique features of this disease and the dramatic public and private sector responses bring these concerns to the forefront. Dying with dignity around those we love is what many would choose if given the chance. However, dying alone in a healthcare setting is not a new phenomenon.

Many find comfort in the perception of having some control over when and how their lives come to a close. One of the factors that makes dying during this time more difficult for patients and their families is the well-founded fear that their loved ones may be alone when they die, regardless of preference or planning. Cleveland Clinic has gone to great lengths to balance the need for public safety with the concern about suffering related to isolation during end-of-life care in patients with COVID-19. This effort, championed by the Office of Patient Experience, includes "compassionate exceptions" to strict visitation policies and extends the provision of PPE to the visitors of these patients. In addition, virtual visitation via the patients' own electronic device (and iPADs if they did not have an electronic device) was rapidly enabled across the healthcare system. Despite these significant efforts, patients likely experience more physical isolation than in pre-COVID times.

The anticipation of grief and suffering related to the death itself is compounded by the suffering isolation induces. This grief may be further exacerbated by understandable and realistic transmission fears that loved ones have when considering being with patients as they die. More than ever, our system is witnessing a stronger desire from patients to leave the hospital as quickly as possible, joining family at home, even if their symptoms are not optimally controlled. Providing education and anticipatory guidance about the normal dying process to patients and family members, when desired, can reduce suffering and help people feel more prepared. ${ }^{9}$

Mitigating distress during this pandemic also comes from aligning treatment plans with patient goals and preferences. Clear and compassionate communication from the healthcare team to the patient's loved ones when a patient's condition changes is also helpful. Prior to COVID-19, patients admitted to the regular nursing floor for respiratory distress related to a chronic or acute condition would have had a conversation with their clinician regarding their care goals and preferences as early as possible in the course of that admission. Considering the unpredictable and rapid changes that can occur with COVID-19, it is imperative these discussions occur at the time of admission for all patients at any level of care. Reasonably, this recommendation extends to ambulatory settings for patients at the time of a COVID-19 diagnosis, especially for those of advanced age or with significant comorbidities. In addition, as we enabled patient engagement platforms to remotely care for patients, advance care planning was integrated into these platforms for patients with COVID-19, and requests for conversation on the topic are responded to by spiritual care.

As we shift among conventional, contingency, and crisis stages of care, ${ }^{10}$ our response must continue to evolve and address ongoing issues related to the allocation of resources. Countries severely affected by COVID-19 in other parts of the world saw overwhelming numbers of deaths, which compromised 
TABLE 1

Summary of the Cleveland Clinic palliative care response plan to the pandemic

Cleveland Clinic Palliative Care Response Plan to COVID-19

Staff

COVID-19 educational resources and tools to facilitate high-quality primary PC by all clinicians

- COVID-19 Comfort Care Electronic Medical Record Order Set

- COVID-19 Symptom Management Guide, Recovery Possible

- COVID-19 Symptom Management Guide, End-of-Life

- Electronic Medical Record Documentation Templates (Progress Note and Death Pronouncement)

- Communication Guide for Advance Care Planning Conversations

- PC Crisis Hotline Pager: 24/7 support available to any caregiver for advice, coaching, or support

Stuff

Ensuring availability of medications and supplies necessary for symptom management and end-of-life care

- Pharmacy and supply chain needs managed by Cleveland Clinic enterprise leadership

Space

Optimizing physical space and facilities

- Cohorting of patients with COVOD-19 in the intensive care unit, and regular nursing floor managed by Cleveland Clinic enterprise leadership

- Consideration of cohorting patients with COVID-19 who are receiving comfort-focused, end-of-life care

- Consideration of freestanding hospice unit capability for patients with and without COVID-19

\section{Systems}

Developing systems to facilitate PC delivery

- Strategic deployment of the specialty PC workforce

- Triage system for specialty PC consultation

- Targeted inpatient consultation staffing plan to maximize collaboration, education, and peer support

- Transition to telehealth platforms to minimize longitudinal care interruption for existing PC patients

- Inclusion of advance care planning conversations in ambulatory monitoring programs for patients with COVID-19

the quality of hospice care delivery. During the surge in Italy, hospice centers were forced to quickly implement practice changes such as shifting from inpatient to community-based care and telephone support. They were further challenged by limits on visitation and variations in the availability of and standard practices for utilization of PPE. ${ }^{11}$

Ongoing monitoring for potential barriers to palliative and hospice care in all settings may facilitate early identification of limitations related to staff, stuff, space, or system-related issues. Fortunately, at this point in the pandemic, local conditions in Cleveland have not led to limitations of medication or supplies. Staffing also remains sufficient. The primary hospice challenge, to date, is a system-related one centered on completing the necessary admission paperwork in a timely fashion. The visitation restrictions and social distancing efforts have had the unintended effect of delaying a patient's transition to a hospice level of care because Medicare Conditions of Participation require patient or family signatures at the time of enrollment. Cleveland Clinic Hospice team members have managed these challenges by leveraging technology or making extra efforts to connect directly with patients' families at locations outside the acute care setting. Additional adaptation of this and other obstructive regulations would facilitate care delivery.

\section{CARING FOR OUR OWN}

Equally important to patient care delivery is the care of our healthcare workers. Those providing or supporting clinical care during this pandemic, especially 
those involved in intensive or end-of-life care, are likely witnessing increased levels of suffering and death, thus heightening their risk of burnout, compassion fatigue, and moral injury. ${ }^{12}$ Support from healthcare system administration and colleagues is imperative. ${ }^{13}$ Social distancing efforts and PPE requirements also impede traditional approaches to collaboration and engagement among healthcare professionals. New alternative and likely virtual approaches to support collaboration and camaraderie for clinical rounds, team huddles, and hand-offs, as well as formal sessions to discuss challenging cases may be helpful. Multiple touchpoints of varying formality offer clinicians opportunities to debrief, discuss feelings, or process unresolved grief with colleagues.

Many clinical teams have already incorporated a variety of these approaches into daily or weekly workflows. The interdisciplinary hospice team at Cleveland Clinic, which includes nurses, chaplains, social workers, physicians and nurse practitioners, performs a daily "pause," which includes a short period of silence to honor patients who have died in the past 24 hours and honor the care provided by the team. Additional support is available through institutional programs such as the Clinic's Caring for Caregivers, the Behavioral Health Department, and the Center for Bioethics.

\section{REFERENCES}

1. Cassel EJ. The nature of suffering and the goals of medicine. N Engl J Med 1982; 306(11):639-645. doi:10.1056/NEJM198203183061104

2. Saunders C. Introduction: history and challenge. In: Saunders C, Sykes N, eds. The Management of Terminal Malignant Disease. Hodder and Stoughton; 1993:1-14.

3. World Health Organization. Integrating palliative care and symp- tom relief into responses to humanitarian emergencies and crises: a WHO guide. https://apps.who.int/iris/handle/10665/274565. Accessed May 11, 2020.

4. Krakauer EL, Daubman B-R, Aloudat T, et al. Palliative care needs of people affected by natural hazards, political or ethnic conflict, epidemics of life-threatening infections, and other humanitarian crises. In: Waldman E, Glass M, eds. A Field Manual for Palliative Care in Humanitarian Crises. Oxford University Press; 2019:chap 2. doi:10.1093/med/9780190066529.003.0002

5. Quill TE, Abernethy AP. Generalist plus specialist palliative care-creating a more sustainable model. N Engl J Med 2013; 368(13):1173-1175. doi:10.1056/NEJMp1215620

6. Arya A, Buchman S, Gagnon B, Downar J. Pandemic palliative care: beyond ventilators and saving lives. CMAJ 2020; 192(15):E400-E404. doi: $10.1503 / \mathrm{cmaj} .200465$

7. Downar J, Seccareccia D. Palliating a pandemic: "all patients must be cared for." J Pain Symptom Manage 2010; 39(2):291-295. doi: 10.1016/j.jpainsymman.2009.11.241

8. Christian, MD, Devereaux AV, Dichter JR, Geiling JA, Rubinson L. Definitive care for the critically ill during a disaster: current capabilities and limitations: from a Task Force for Mass Critical Care summit meeting, January 26-27, Chicago, IL. Chest 2008; 133 (5 suppl): 8S-17S. doi: 10.1378/chest.07-2707

9. Wallace $C L$, Wladkowski SP, Gibson A, White P. Grief during the COVID-19 pandemic: considerations for palliative care providers. J Pain Symptom Manage 2020; April 13. doi:10.1016/j. jpainsymman.2020.04.012

10. Maves RC, Downar J, Dichter JR, et al. Triage of scarce critical care resources in COVID-19- an implementation guide for regional allocation: an expert panel report of the Task Force for Mass Critical Care and the American College of Chest Physicians. Chest 2020; April 11. doi:10.1016/j.chest.2020.03.063

11. Costantini M, Sleeman KE, Peruselli C, Higginson IJ. Response and role of palliative care during the COVID-19 pandemic: a national telephone survey of hospices in Italy. Palliat Med 2020; April 29. doi:10.1177/0269216320920780

12. Doherty M, Hauser J. Care of the dying patient. In: Waldman E, Glass M, eds. A Field Manual for Palliative Care in Humanitarian Crises. Oxford University Press; 2019:chap 9. doi:10.1093/ $\mathrm{med} / 9780190066529.003 .0009$

13. Hendin A, La Rivière CG, Williscroft DM, O'Connor E, Hughes J, Fischer LM. End-of-life care in the emergency department for the patient imminently dying of a highly transmissible acute respiratory infection (such as COVID-19). CJEM 2020; March 26. doi:10.1017/cem.2020.352. 